\title{
Nonlinear analysis of the degree of order and chaos of morphology of porous silicon nanostructures
}

\author{
Z Zh Zhanabaev ${ }^{1,2,3}$, T Yu Grevtseva ${ }^{1,2,3}$, K A Gonchar ${ }^{4}$, G K Mussabek ${ }^{1,2,5}$, \\ D Yermukhamed $^{1,2}$, A A Serikbayev ${ }^{2}$, R B Assilbayeva ${ }^{6}$, \\ A Zh Turmukhambetov ${ }^{2}$ and $\mathrm{V}$ Yu Timoshenko ${ }^{4,5}$
}

\begin{abstract}
${ }^{1}$ National Nanotechnological Laboratory of Open Type at al-Farabi Kazakh National University, al-Farabi av., 71, Almaty, Kazakhstan, 050040

${ }^{2}$ Al-Farabi Kazakh National University, al-Farabi av., 71, Almaty, Kazakhstan, 050040

${ }^{3}$ Institute of Experimental and Theoretical Physics, al-Farabi av., 71, Almaty, Kazakhstan, 050040

${ }^{4}$ Lomonosov Moscow State University, Leninskie Gory, 1, Moscow, Russia, 119991

${ }^{5}$ National Research Nuclear University "MEPhI", Institute of Engineering Physics for Biomedicine, Laboratory “Bionanophotonics”, Kashirskoe sh., 31, Moscow, Russia, 115409

${ }^{6}$ Caspian State University of Technology and Engineering named after Sh. Yessenov, 30 microdistrict, 14/2, Aktau, Kazakhstan, 130000
\end{abstract}

e-mail:mptl449a@gmail.com, serikbayev.almas@gmail.com

\begin{abstract}
This work has been done to identify quantitative criteria the degree of order and chaos morphology of porous layers consisting of silicon nanowire arrays. In order to fulfill the work, a method of using metal-assisted chemical etching has been utilized to produce nanowires. There has been done a work of digital processing of porous film images which were extracted by scanning electron microscope. Informational-entropic and Fourier analysis have been applied to quantitatively describe the degree of order and chaos in nanostructure distribution in the layers. Self-similarity of the layer morphology has been quantitatively described via its fractal dimensions by correlation method. The applied approach for image processing allows us to distinguish the morphological features of as-called "black" (more ordered) and "white" (less ordered) silicon layers, which are characterized by minimal and maximal optical reflection, respectively. From all of the methods of digital techniques that we have used the method for determining the conditional information of a chaotic set was proved to be the most informative.
\end{abstract}

\section{Introduction}

Recently porous silicon (Si) nanostructures as nanowires (NWs) are intensively studied in view of their possible applications in optoelectronics, photonics, and sensorics [1-5]. Si NWs possess unique electrical and optical properties, which are prospective for biomedicine [6-8] and advanced energy and environment applications [9]. Porous silicon films are of particular interest because the electrical and optical properties of these films depend on their porosity, thickness, size distribution of pores and nanocrystals. In general, a tailoring of the nanostructure morphology seems to be promising for the development of new semiconductor devices for different purposes. 
Nanocluster semiconductor films, including porous layers of silicon nanowire (SiNWs) grown in non-equilibrium conditions have scale-invariant, hierarchically self-similar, i.e. fractal and multifractal structure [10-13]. The scale invariance of a SiNW film is in their self-similarity (similarity coefficients on different variables are equal to each other) and self-affinity (similarity coefficients are different on different variables, that corresponds to anisotropic structure of the film).

Fractal distribution of nanoclusters and pores in a film, which is grown by wet chemistry method, can be caused by processes of self-organization occurred in non-equilibrium non-linear open systems. The self-organization is considered as appearance of the order from chaos. Description of porous silicon films with partially ordered SiNWs is an interesting scientific problem because the informational entropy and fractal dimension can be used as quantitative characteristics of dynamical chaos. According to the well-known Prigozhin theorem, derivative of entropy with respect to time tends to its minimal value at self-organization. In agreement with Klimontovich's S-theorem [14], entropy decreases at self-organization of a system. Possibility to define the entropy via fractal measure has been described in Ref.[15]. It is noted, that if the order of multifractal moment characterizing a hierarchical system is equal to unity, one can calculate entropy of the system. However, calculation of multifractal moment's order is a stand-along scientific problem. The ordering of different complex systems (universe, galaxies, oscillatory systems, etc.) on different spatial scales also can be described using entropy [16-18]. The informational entropy can be considered as a value containing information about the system [16]. The thermodynamic entropy can be used for the description of organized structures evolution, for example, expansion of Universe [17]. In Ref.[18] it is shown that thermodynamic entropy can be used for control of self-organized criticality by description of nanosized structure of thin film coatings.

The informational entropy is a basic building block of complexity theory including theories of chaos and fractals $[19,20]$. To evaluate the informational entropy of nanostructured systems it is required more accurate analysis of surface and bulk nano-thermodynamics. The problem is in the fact that for the description of equilibrium systems, usually Boltzmann entropy is used. For the description of non-equilibrium systems one should consider the Shannon entropy. But in this case we have difficulties related with normalization of entropy, because entropy tends to infinity at decreasing of size of cells to zero. In this paper we will pay attention to this problem.

Fractal analysis of images allows us to detect and describe singularities of cluster structure of films. The fractal analysis is useful for the description of self-affinity of films with different chemical composition and for quantitative description of surface waviness, irregularity, roughness and anisotropy [21]. Calculation of the fractal dimensions of different surface can be used for their optimal position at grinding processes [22].

As usual, distribution of pores in different materials is characterized by fractal regularities. Studies of the relation between fractal dimension and porosity have been described in Refs. [23-25]. Thus, the fractal dimension of porous membranes significantly depends on percentage ratio of components [23]. Calculations of numeric values of the fractal dimension of porous samples are used in computerized tomography for the description of pore structure of rocks [24]. An analysis of the porosity and pore structures by using the fractal and multi-fractal approaches is widely used in geology for oil, gas and geothermal systems [25]. However, in general, the desired relationship between porosity and fractal dimension is ambiguous. Objects containing fractals with different number of iterations of their parts (prefractals) have the same values of fractal dimension but different values of porosity. Note that a description of the physical processes in nanostructures with quantum properties is possible on the base of comprehensive analysis of their scale-invariant (fractal), informational-entropic, topological, and spectral characteristics. The present work is aimed to quantitatively describe the scale-invariant structure of porous SiNWs layers and to develop an adequate technique for distinguishing films of "black" and "white" silicon by their morphology.

\section{Experimental}

Samples of two types, i.e. so-called "black" and "white" porous layers, were obtained by metalassisted chemical etching (MACE), which is a "top-down" approach of material processing by dissolution catalyzed with noble metal nanoparticles [6-9]. As a substrate we used (100) oriented p- 
type c-Si wafers with resistivity of $1-10 \Omega^{*} \mathrm{~cm}$ and the treatment was carried out in three stages: 1 ) deposition of catalyst metal particles on the substrate surface, 2) chemical etching of the substrate and 3) removal of residual metal particles. In our experiments as the catalyst we used silver (Ag) nanoparticles, which were precipitated on the surface of c-Si substrates from a mixture of $0.02 \mathrm{M}$ aqueous solution of AgNO3 and 5M aqueous solution of hydrofluoric acid (HF) in the volume ratio 1:1 for $45 \mathrm{sec}$. Then the MACE treatment was done by immersing the samples in a mixture of 5M HF and $30 \%$ hydrogen peroxide (H2O2) taken in the volume ratio of 10:1. The length of SiNWs (layer thickness) was determined by the etching time. To obtain samples of "black silicon" and "white silicon" the MACE treatment was performed during 1-10 minutes and 0.5-6 hours, correspondingly. After the MACE process, the samples were immersed in 45\% concentrated nitric acid (HNO3) for 15 minutes to remove residual Ag particles and then the samples were washed in de-ionized water and dried in air.

The structure properties of the obtained samples were studied by means of the scanning electron microscopy (SEM) using an ULTRA 55 FE-SEM (Carl Zeiss) microscope. Spectra of the total reflection were measured in the optical range from 0.2 to $1.2 \mu \mathrm{m}$ using a spectrophotometer Lambda 35, Perkin Elmer. The optical measurements were carried out at room temperature in air.

Typical spectra of the total reflectance of SiNW arrays with low ("black") and high ("white") level of the reflectance are shown in Figure 1.

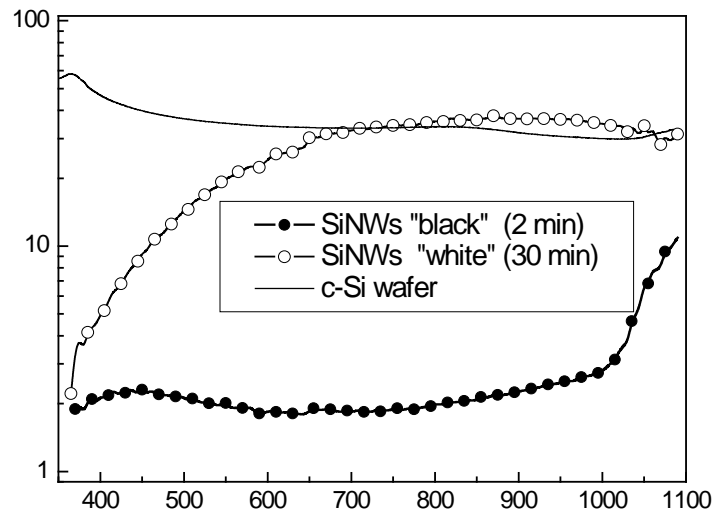

Figure 1. Total reflection spectra of two samples of SiNWs prepared for 2 min (close circles) and 30 min (open circles) as well as for a double-side polished c-Si wafer (solid line).

\section{Analysis of SEM images}

\subsection{Porosity evaluation}

Porosity of a SiNW layer can be experimentally determined by gravimetric measurements of the corresponding substrate before and after MACE [7,8]. However, this method is characterized by relatively low accuracy when the mass measurement occurred at a nanoscale. While the porosity of a thin film of SiNWs can be calculated from its optical density [6], it gives only the average porosity and it can be only applied for films with low light scattering.

Different methods can be used for image processing [26-28]. In our work the porosity of the top of SiNW layer was estimated from an analysis of the corresponding top view SEM images. The analysis was done in the following way. At the first stage a square part of a SEM image with sizes $700 \times 700$ pixels was selected (see for example Figure 2a). At the second step, the selected area (Figure 2(b)) has been subjected to conversion of contrast enhancement. At the third step, the image was converted to the "black and white" format (Figure 2(c)). The corresponding histogram of the brightness distribution of pixel is shown in Figure 2(d). The horizontal axis corresponds to the brightness level, $B$, in the range from 0 (black pixels) to 255 (white pixels), and the vertical axis represents number $N$ of pixels.

Porosity of the films was calculated as follows 


$$
\eta=1-\frac{N_{w}}{N_{w}+N_{b}},
$$

where $N_{b}$ and $N_{w}$ are numbers of black (pores) and white (Si nanocrystal) pixels, correspondently.

a)
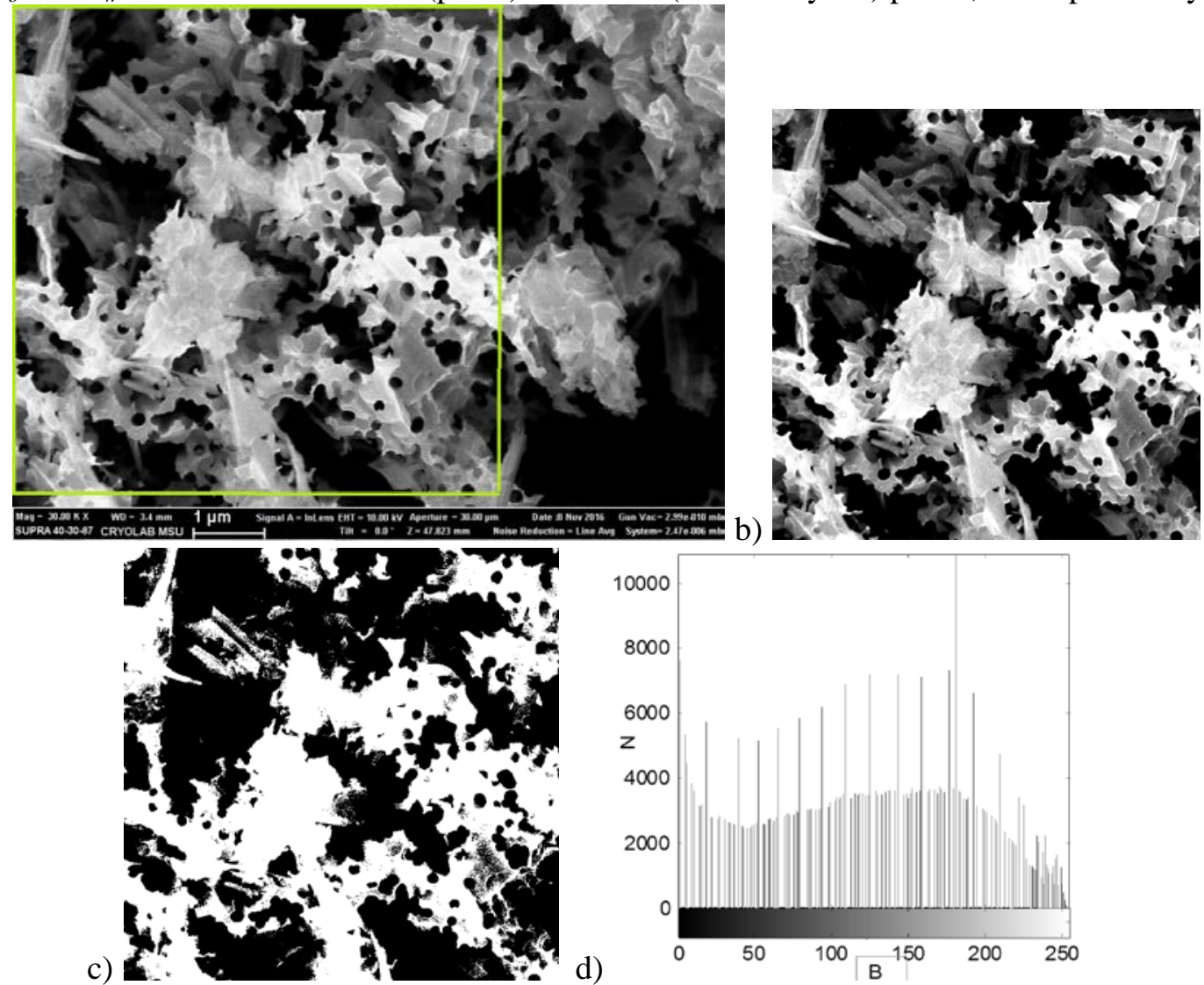

Figure 2. a) SEM image of a porous Si film where green lines select a fragment for the digital analysis; (b) the fragment selected for image processing; (c) the segment image with high contrast containing only white and black pixels; (d) histogram of the distribution of pixel intensities in the image.

\subsection{Information entropy analysis}

SEM images of investigated silicon films show that these films have porous structure and contain sets of quantum nanowires with complex internal structure. These sets form separated clusters with different shape and chaotic distribution. The information entropy is widely used to characterize the chaotic state of an object. We have defined its numeric value via the following well-known formula:

$$
S(x, y)=-\sum_{i=1}^{N} \sum_{j=1}^{N} P_{i, j}(x, y) \ln P_{i, j}(x, y),
$$

where $P_{i, j}$ is the probability of pixel with a certain brightness proportional to histogram counts (Figure 2(d)), which correspond to a segment of the original image in $(x, y)$ plane.

Dependence of non-normalized informational entropy on porosity of the films is shown in Figure 3. In case of using the expression $S(x, y) /(S(x)+S(y))$ we obtain values of entropy normalized to unit because entropy is maximal if a process is independent on variables $x$ and $y$. Surfaces of "white" silicon observed in the vertical direction (top view) have bigger values of porosity than lateral sides of the films. The entropy of a top side of film decreases with increasing of porosity, but entropy of its lateral side increases. It should also be noted that entropy of "black" silicon films is smaller than entropy of "white" silicon films by about 50\%. It means that "black" silicon is more ordered than 
"white" silicon, i.e. pore sizes are distributed according to some regularity and coherent absorption of photons is possible

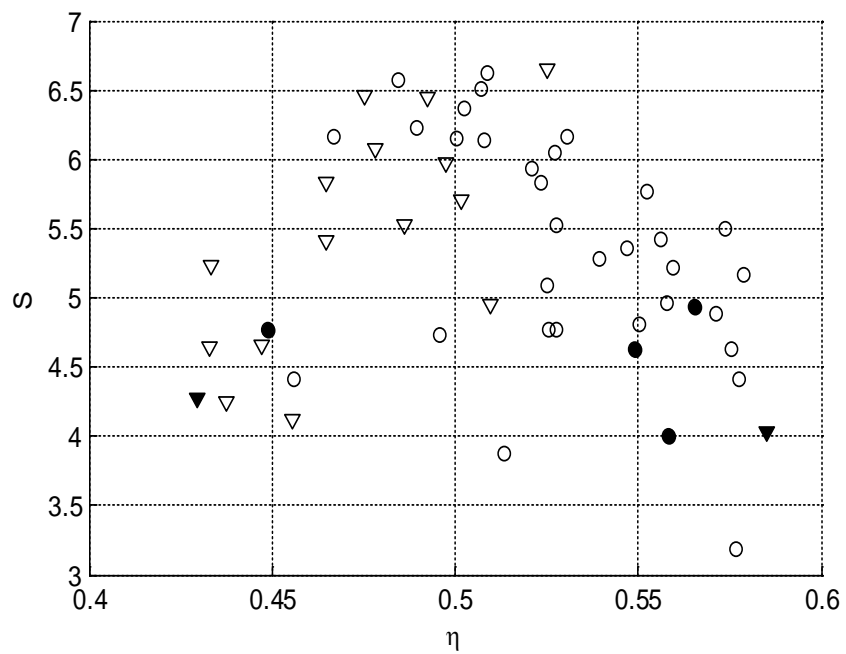

Figure 3. Dependence of the information entropy on porosity of films.

Number of cells (pixels is $700 \times 700$ )

$$
\begin{aligned}
& \text { o - "white" silicon (top view); } \\
& \nabla \text { - "white" silicon (lateral view); } \\
& \bullet \text { - "black" silicon (top view); } \\
& \boldsymbol{\nabla} \text { - "black" silicon (lateral view). }
\end{aligned}
$$

Figure 4 represents a dependence of the information-to entropy ratio (IER) on porosity. The information $(I(x \mid y))$ has been defined as a difference between full entropy $\mathrm{S}(\mathrm{x}, \mathrm{y})$ and conditional entropy $S(x \mid y)$ as

$$
I(x \mid y)=S(x, y)-S(x \mid y)
$$

where $x, y$ are horizontal and vertical coordinates.

The designation $I(x \mid y)$ corresponds to values of information calculated via variable $x$ at known value of $y$. Entropy $S(x \mid y)$ can be defined via conditional probability as $P(x \mid y)=P(x, y) / P(y)$.

Formula (3) reflects the generally accepted definition of information which meaning is measure of order (certainty) [14]. Conditional entropy (corresponding to some order) is always less then unconditional entropy (absence of order), so, $I(x \mid y)$ is always greater than zero. In the theory of telecommunications formula (3) contains $S(x)$ instead of $S(x, y)$. It leads to understated values of $I(x \mid y)$.

The relation of $I(x \mid y) / S(x \mid y)=I E R$ (information-to-entropy ratio) is an analog of signal-to-noise ratio $S N R$ widely used in radiophysics [29-31]. Difference between these values is in the fact that $S N R$ should be calculated at a known noise level, but IER can be defined without knowing the noise level. While the entropy of "black" silicon is less than that of "white" one, the information is larger.

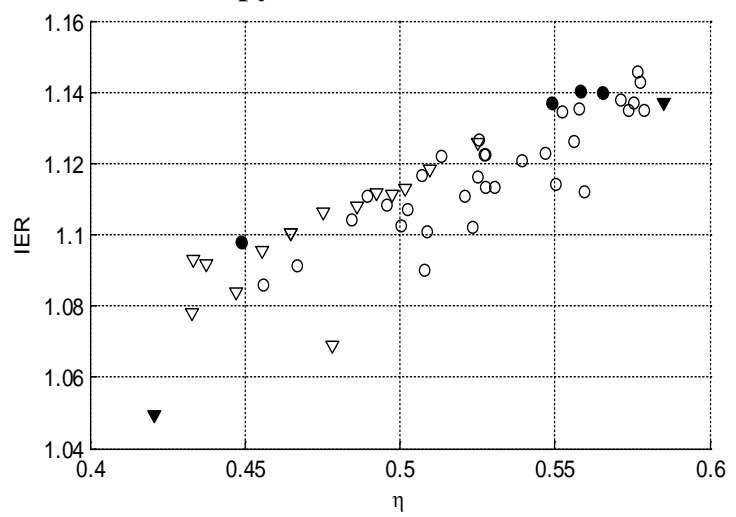

Figure 4. Dependence of the information-to-entropy ratio on porosity of SiNW films

O - "white" silicon (top view);

$\nabla$ - "white" silicon (lateral view);

- - "black" silicon (top view);

$\boldsymbol{\nabla}$ - "black" silicon (lateral view).

\subsection{Fractal dimension analysis}

Fractal dimensions of the films have been defined by use of the box-counting method. As expected, due to scale-invariant structure of nanostructured films values of their fractal dimensions differ insignificantly (the values belong to the range $1.80 \div 1.95$ ). Although values of fractal dimension vary insignificantly because of presence of prefractals (fractals of different iterations), porosity can vary significantly. This fact is evident from Figure 5 illustrating dependence of the fractal dimension on porosity of the films. 


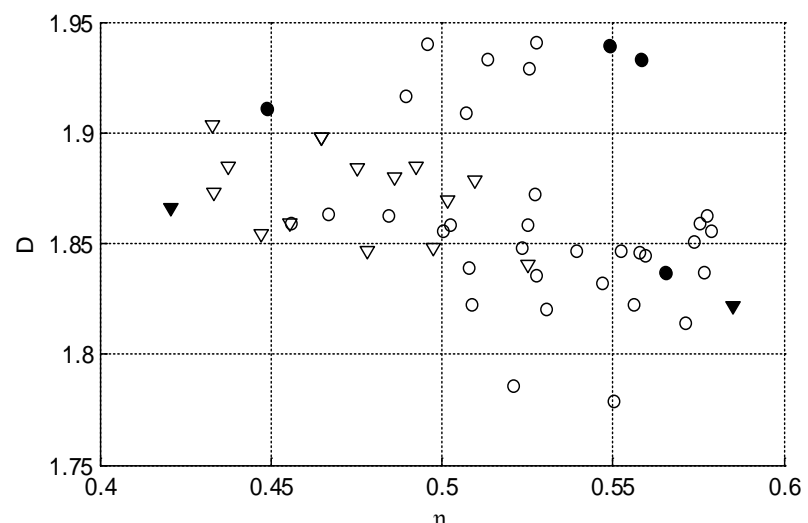

Figure 5. Dependence of fractal dimension of SiNW films on their porosity.

O - "white" silicon (top view);

$\nabla$ - "white" silicon (lateral view);

- - "black" silicon (top view);

$\boldsymbol{\nabla}$ - "black" silicon (lateral view).

a)
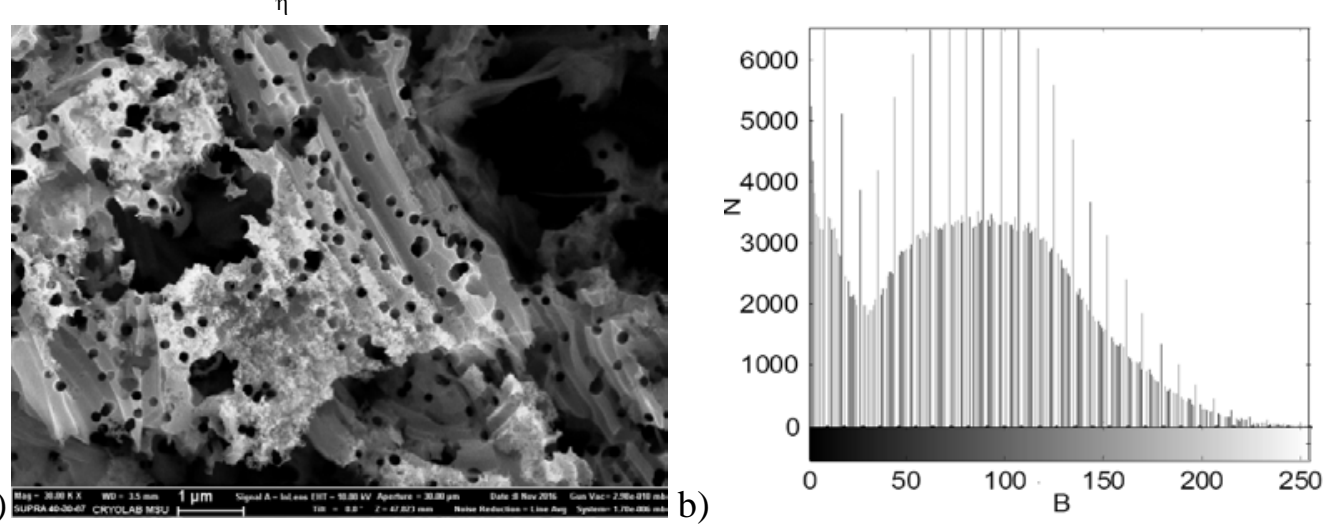

c)

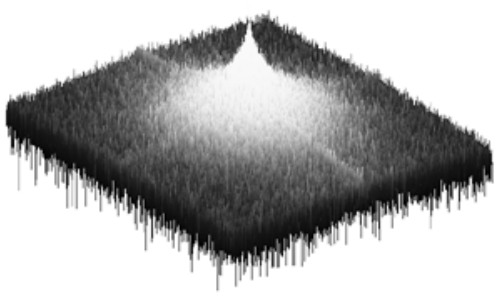

d)
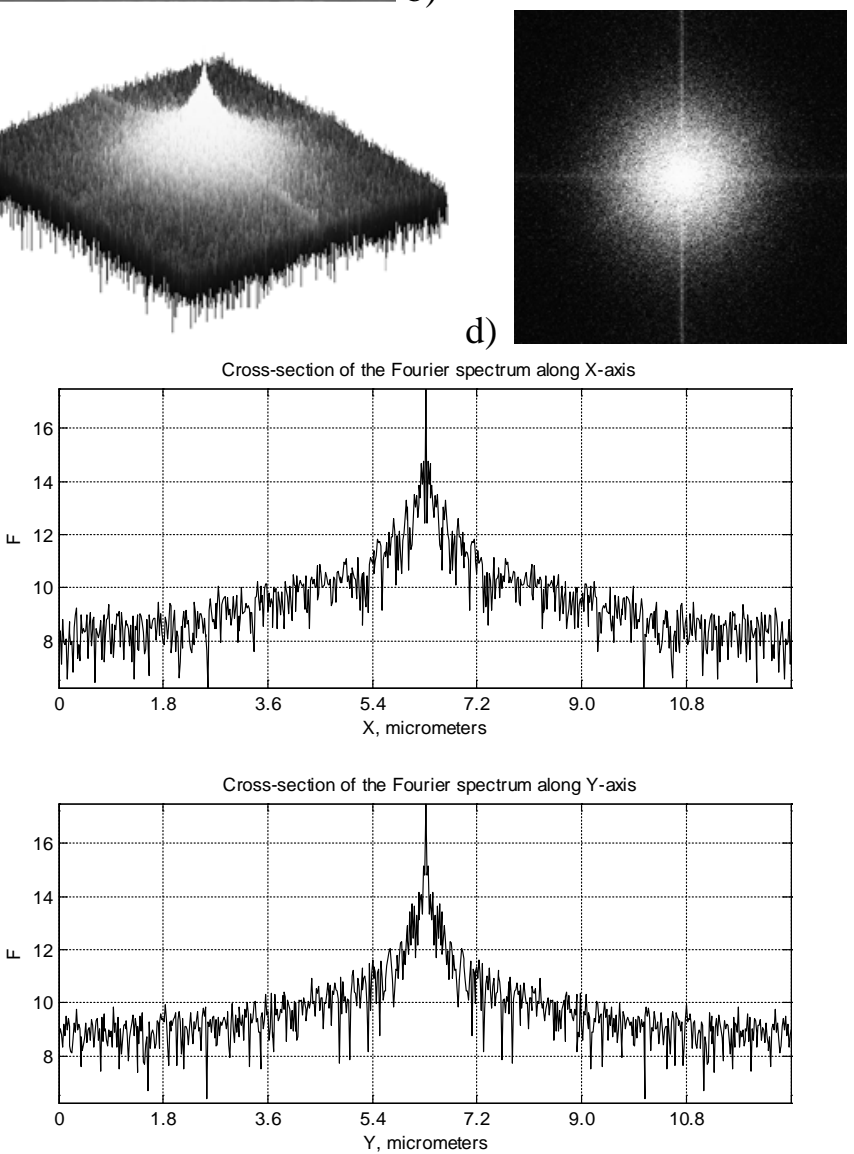

e)

Figure 6. SEM image of "white" silicon film (top view) (a), histogram of pixel intensities of the image (b), three-dimensional Fourier spectrum (c), projection of the Fourier spectrum on the plane (d), cross-section of three-dimensional spectrum along X- and Y-axes (e). 


\subsection{Fourier analysis of SEM-images}

Results of an application of the two-dimension Fourier transform analysis for the description of SEM images of different films are shown in Figures. 6-9. Figure 6 illustrates results obtained at processing of a typical image of "white" silicon film (top view). Figures 7, 8 and 9 describe "white" silicon (lateral view), "black" silicon (top view) and "black" silicon (lateral view), correspondently. The Fourier transform has been applied to elements of matrix describing pixel intensities of an original image. Cross-section lines of three-dimensional Fourier spectra are drawn along the abscissa and ordinate axes in such way that they pass through centers of the graphs. Using two-dimensional Fourier transform let us describe type of silicon films ("black" or "white" silicon). Weak asymmetry in the Fourier spectra corresponds to certain anisotropy of structure of the films caused by experimental conditions.

a)

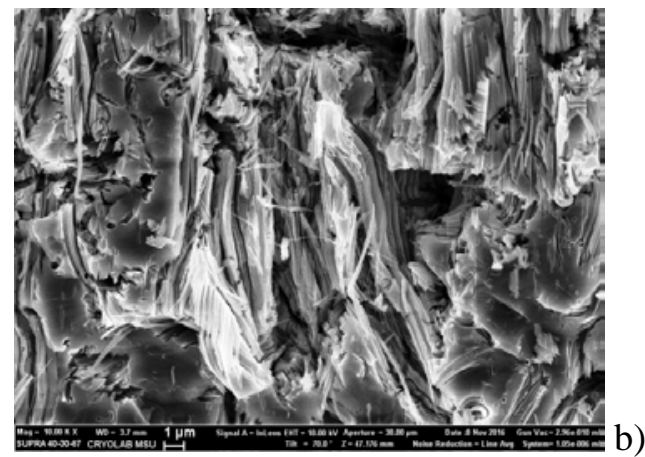

c)

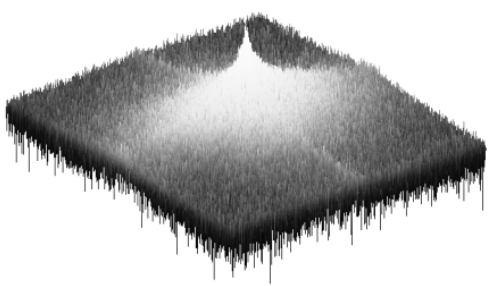

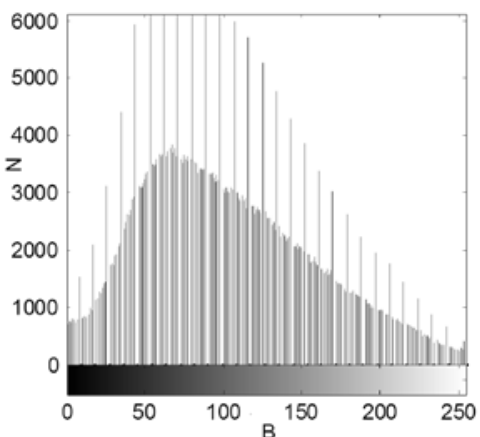

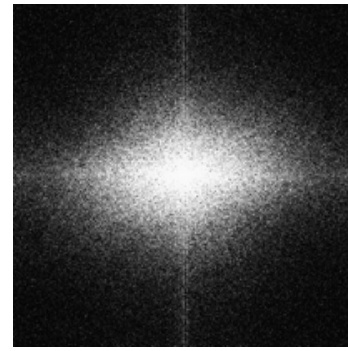

d)

Cross-section of the Fourier spectrum along $\mathrm{X}$-axis

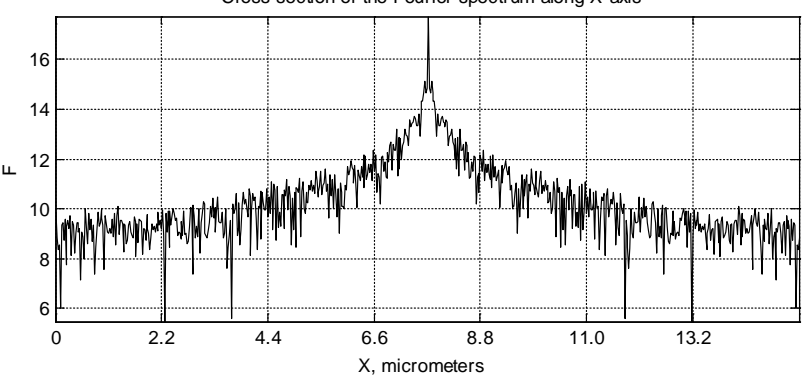

Cross-section of the Fourier spectrum along $\mathrm{Y}$-axis

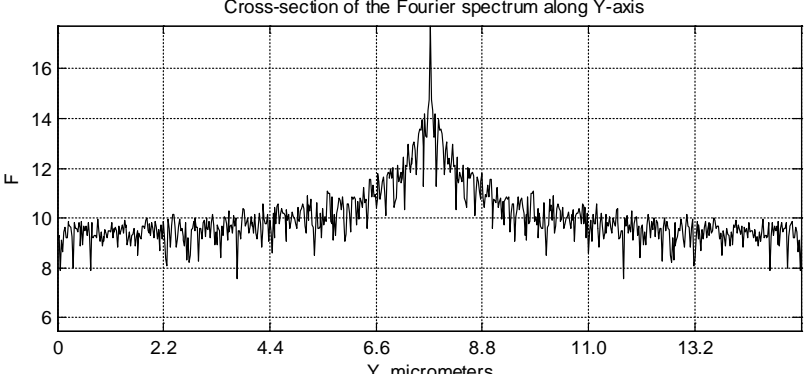

e)

Figure 7. Cross-sectional SEM image of "white" silicon film (lateral view) (a), histogram of pixel intensities of the image (b), three-dimensional Fourier spectrum (c), projection of the Fourier spectrum on the plane (d), cross-section of three-dimensional spectrum along X-and Y-axes (e). 
From the presented above graphs we can see that histograms describing distribution of pixel intensities of images of "white" and "black" silicon films are noticeably different: histograms corresponding to "white" silicon are usually solid, but histograms of "black" silicon contain sharp bursts. This difference between histograms of "white" and "black" silicon films indicate to the fact that "black" silicon has more expressed structuredness. These characteristic features of the histograms can be used for classification of silicon films to "white" and "black" silicon.

a)

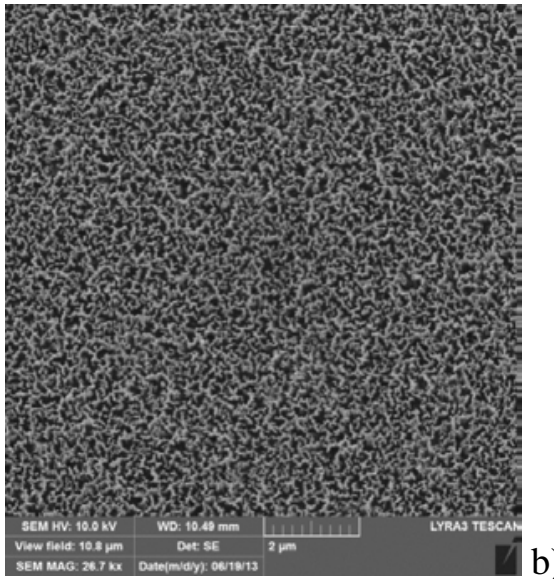

c)

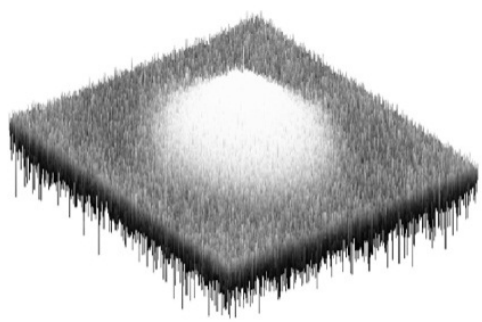

d)
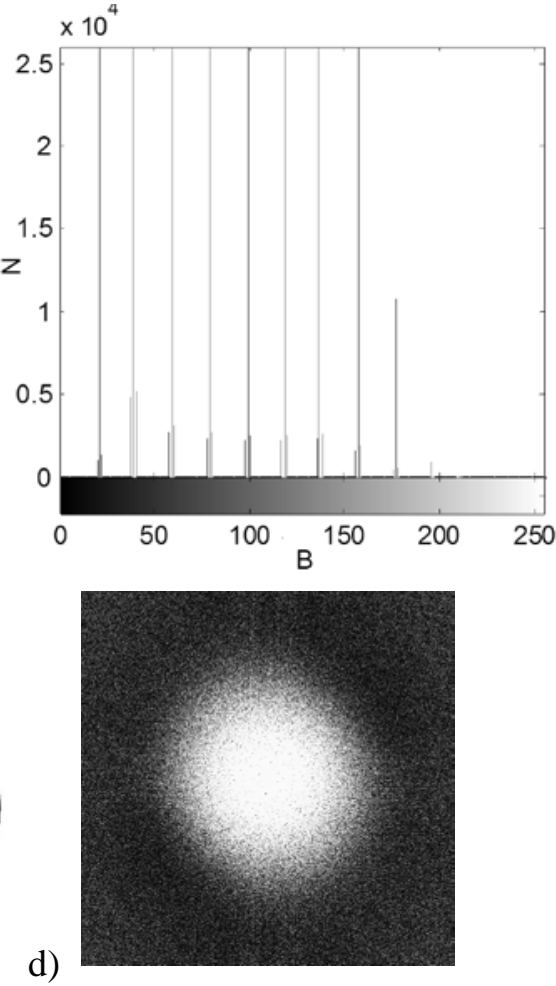

Cross-section of the Fourier spectrum along $\mathrm{X}$-axis

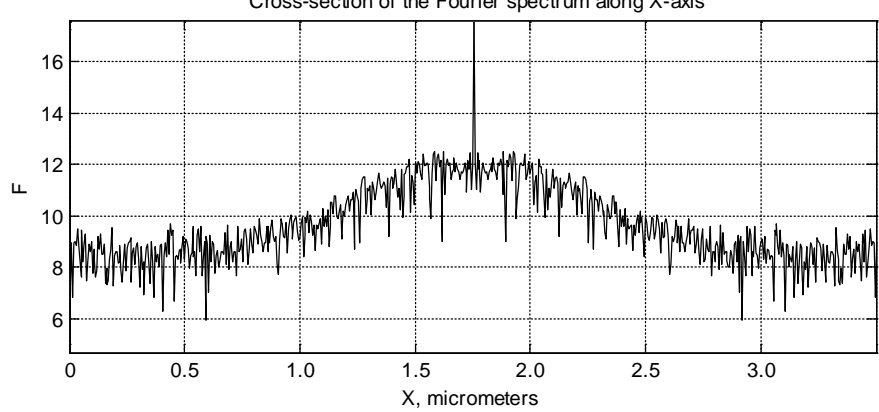

Cross-section of the Fourier spectrum along $\mathrm{Y}$-axis

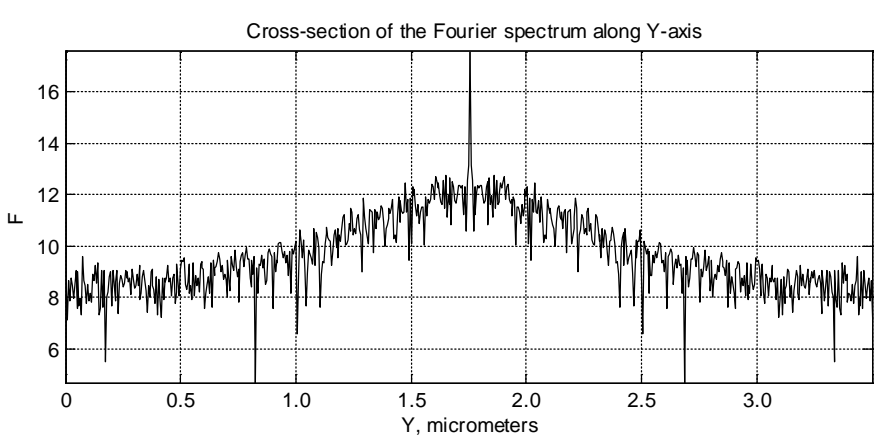

e)

Figure 8. Image of "black" silicon film (top view) (a), histogram of pixel intensities of the image (b), three-dimensional Fourier spectrum (c), projection of the Fourier spectrum on the plane (d), crosssection of three-dimensional spectrum along $\mathrm{X}$ - and $\mathrm{Y}$-axes (e). 
a)

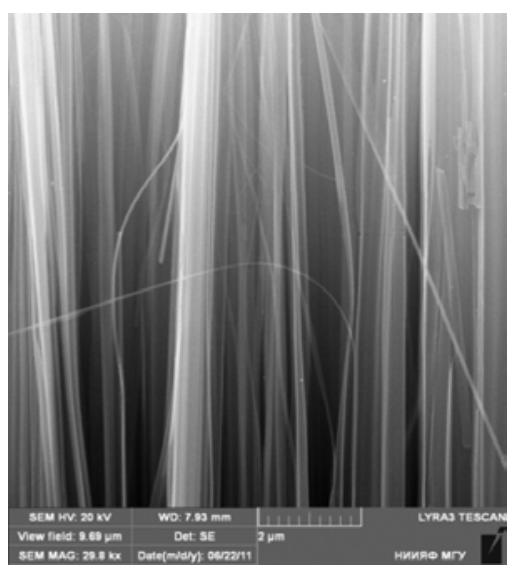

c)

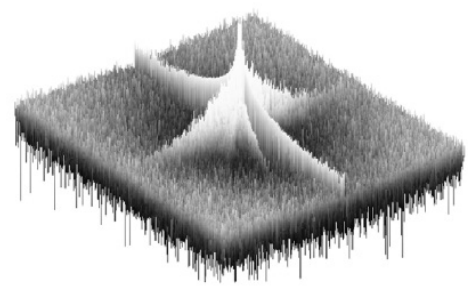

d)
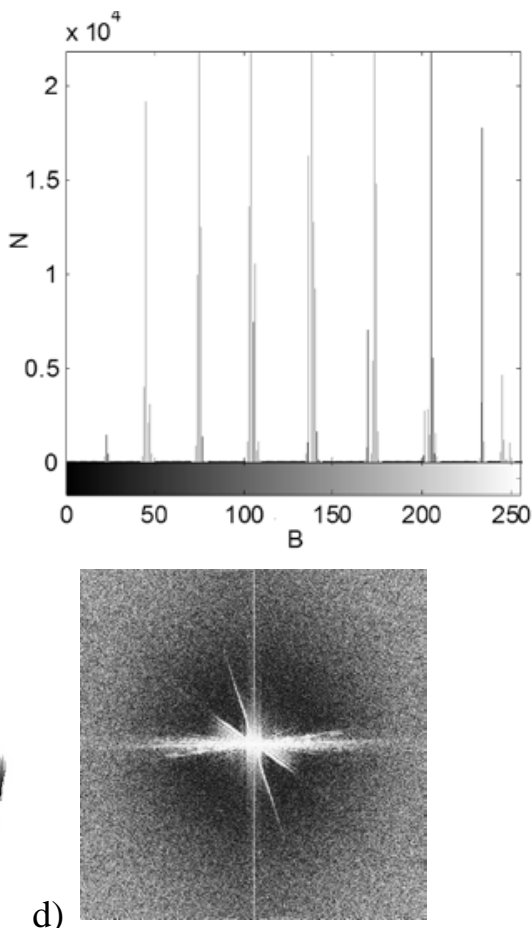

Cross-section of the Fourier spectrum along X-axis

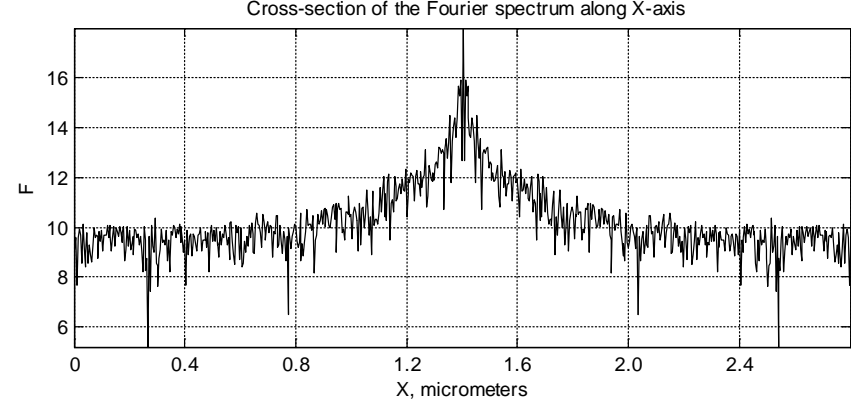

Cross-section of the Fourier spectrum along $\mathrm{Y}$-axis

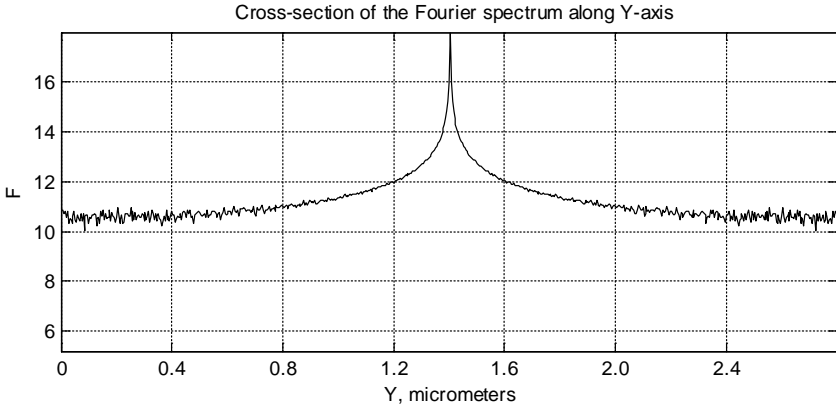

e)

Figure 9. Cross-sectional SEM mage of "black" Si film (lateral view) (a), histogram of pixel intensities of the image (b), three-dimensional Fourier spectrum (c), projection of the Fourier spectrum on the plane (d), cross-section of three-dimensional spectrum along X-and Y-axes (e).

\section{Conclusions}

The quantitative analysis of SEM images of nanostructured films with properties of "white" and "black" silicon, which were formed by MACE c-Si wafers, allowed us to estimate the porosity varied from $42 \%$ to $53 \%$ for their lateral sides and from $46 \%$ to $58 \%$ for their top sides. Thus, the top surfaces of "white" silicon have larger porosity than that for the lateral sides and this fact indicates the gradient of morphology related to the MACE growth of Si NWs accompanied with their gradual chemical dissolution. The revealed difference between information entropy for "black" and "white" Si films shows that the structure of the former is more ordered than that for the latter. The fractal 
dimensions of the both types of nanostructured Si layers are different due to the presence of fractals with different iterations. The Fourier analysis of SEM images also indicates that the "white" silicon films are more isotropic than the "black" ones. This fact is confirmed by values of the scaling factor describing colored noise typical for distribution of nanostructures. The distribution histogram of pixel intensities in the SEM images of the top of Si NW arrays reveals the Gaussian function and a power law for the "white" and "black" samples, respectively. Thus, the performed informational-entropic, fractal, spectral, and statistical treatments of the SEM images indicate that the optical properties of "black" and "white" samples are related to the more ordered structure of the former that ensures the stronger effective absorption of light with photon energies below the bandgap.

\section{References}

[1] Jarimaviciute-Zvalionienea R, Prosycevasa I, Kaminskienea Z and Lapinskas S 2011 Optical properties of black silicon with precipitated silver and gold nanoparticles Acta Physica Polonica A 120 942-945

[2] Koynov S, Brandt M S and Stutzmann M 2006 Black nonreflecting silicon surfaces for solar cells Applied Physics Letters 88203107

[3] Lua Y T and Barronw A R 2013 Nanopore-type black silicon anti-reflection layers fabricated by a one-step silver-assisted chemical etching Phys. Chem. Chem. Phys. 15 9862-9870

[4] Ravindra N M, Marthi S R and Sekhri S 2015 Modeling of Optical Properties of Black Silicon/Crystalline Silicon Journal of Scientific and Industrial Metrology 1(1) 1-7

[5] Ge M, Rong J, Fang X and Zhou Ch 2012 Porous doped silicon nanowires for lithium ion battery anode with long cycle life Nano Letters 12 2318-2323

[6] Osminkina L A, Sivakov V A, Mysov G A, Georgobiani V A, Natashina U A, Talkenberg F, Solovyev V V, Kudryavtsev A A and Timoshenko V Yu 2014 Nanoparticles prepared from porous silicon nanowires for bio-imaging and sonodynamic therapy Nanoscale Research Letters 9(463) $1-7$

[7] Gonchar K A, Osminkina L A, Galkin R A, Gongalsky M B, Marshov V S, Timoshenko V Yu, Kulmas M N, Solovyev V V, Kudryavtsev A A and Sivakov V A 2012 Growth, structure and optical properties of silicon nanowires formed by metal-assisted chemical etching Journal of Nanoelectronics and Optoelectronics 7(6) 602-606

[8] Crescentini M, Rossi M, Ashburn P, Lombardini M, Sangiorgi E, Morgan H and Tartagni M 2016 AC and Phase Sensing of Nanowires for Biosensing Biosensors 6(15) 1-14

[9] Ghosh R and Giri P K 2017 Silicon nanowire heterostructures for advanced energy and environmental applications: a review Nanotechnology 28012001

[10] Zhanabaev Z Zh, Grevtseva T Yu, Danegulova T B and Assanov G S 2013 Optical processes in nanostructured semiconductors Journal of Computational and Theoretical Nanoscience 10(3) 673-678

[11] Zhanabaev Z Zh and Grevtseva T Yu 2014 Physical fractal phenomena in nanostructured semiconductors Reviews in Theoretical Science 2(3) 211-259

[12] Zhanabaev Z Zh, Grevtseva T Yu and Ibraimov M K 2016 Morphology and electrical properties of silicon films with vertical nanowires Journal of Computational and Theoretical Nanoscience 13 615-618

[13] Fazio B, Artoni P, Iatì M A, D’Andrea C, Faro M J L, Sorbo S D, Pirotta S, Gucciardi P G, Musumeci P, Vasi C S, Saija R, Galli M, Priolo F and Irrera A 2016 Strongly enhanced light trapping in a two-dimensional silicon nanowire random fractal array Light: Science \& Applications 5 1-7

[14] Klimontovich Yu L 1998 Information Concerning the States of Open Systems Physica Scripta 58(6) 549-555

[15] Slomczynski W, Kwapier J and Zyczkowski K 2000 Entropy computing via integration over fractal measures Chaos 10(1) 180-188

[16] Hwang W Y 2014 A coherent view on entropy Natural Science 6 540-514

[17] Frautschi S 1982 Entropy in an expanding universe Science 217(4560) 593-599 
[18] Fox-Rabinovich G, Paiva J M, Gershman I, Aramesh M, Cavelli D, Yamamoto K, Dosbaeva G and Veldhuis S 2016 Control of self-organized criticality through adaptive behavior of nanostructured thin film coatings Entropy 18290

[19] Gao J, Liu F, Zhang J, Hu J and Cao Y 2013 Information entropy as a basic building block of complexity theory Entropy $153396-3418$

[20] Dezso A and Kaptay G 2017 On the Configurational Entropy of Nanoscale Solutions for More Accurate Surface and Bulk Nano-Thermodynamic Calculations Entropy 19248

[21] Khamesee M B, Kurosaki Y, Matsui M and Murai K 2004 Nanofractal analysis of material surfaces using atomic force microscopy Materials Transactions 45(2) 469-478

[22] Prabhu S and Vinayagam B K 2011 Fractal dimensional surface analysis of AISI D2 tool steel material with nanofluids in grinding process using atomic force microscopy Journal of the Brazilian Society of Mechanical Sciences and Engineering 33(4) 459-466

[23] Wei Q and Wang D. 2003 Pore surface fractal dimension of sol-gel-derived Al2O3-SiO2 membranes Materials Letters 57 2015-2020

[24] Peng R D, Yang Y C, Ju Y, Mao L T and Yang Y M 2011 Computation of fractal dimension of rock pores based on gray CT images Chinese Sci Bull 56(31) 3346-3357

[25] Anovitz L M and Cole D R 2015 Characterization and analysis of porosity and pore structures Reviews in Mineralogy \& Geochemistry 80 61-164

[26] Gashnikov M V 2017 Minimizing the entropy of post-interpolation residuals for image compression based on hierarchical grid interpolation Computer Optics 41(2) 266-275 DOI: 10.18287/2412-6179-2017-41-2-266-275

[27] Vizilter Y V, Gorbatsevich V S, Vishnyakov B V and Sidyakin S V 2017 Object detection in images using morphlet descriptions Computer Optics 41(3) 406-411 DOI: 10.18287/2412-61792017-41-3-406-411

[28] Myasnikov V V 2018 Description of images using a configuration equivalence relation Computer Optics 42(6) 998-1007 DOI: 10.18287/2412-6179-2018-42-6-998-1007

[29] Seshadri R and Penchalaiah N 2011 Method for reducing of noise by improving signal-to-noiseratio in wireless Lan International Journal of Network Security \& Its Applications 3(5) 115-120

[30] Naylor G and Johannesson R B 2009 Long-term signal-to-noise ratio at the input and output of amplitude-compression systems Journal of the American Academy of Audiology 20 161-171

[31] Abbott B P 2016 Observation of gravitational waves from a binary black hole merger Physical Review Letters $116061102-061116$

\section{Acknowledgments}

This work was partially supported by the Committee of Science of the Ministry of Education and Science of the Republic of Kazakhstan (Grant AP05132854, Grant AP05132738). G.K.M. and V.Yu.T. acknowledge the support of the Comprehensive Program of NRNU "MEPhI". 